Adaptive Emotion Regulation, Academic Performance, and

Internalising Problems in Flemish Children with Special Educational

Needs: A Pilot Study

Henk Weymeis ${ }^{\mathbf{a}}$, Karla Van Leeuwen ${ }^{\mathbf{b}}$, \& Caroline Braet ${ }^{\mathbf{a}}$

${ }^{a}$ Department of Developmental, Personality, and Social Psychology Department, Ghent

University, Henri Dunantlaan 2, 9000, Ghent, Belgium

${ }^{b}$ Parenting and Special Education Research Unit, KU Leuven, Leopold

Vanderkelenstraat 32 - bus 3765, 3000, Leuven, Belgium

Henk.Weymeis@UGent.be, Karla.Vanleeuwen@kuleuven.be,

Caroline.Braet@UGent.be

Please address all correspondence to: Henk Weymeis

Acknowledgements: The authors thank 'MPI 't Craeneveld' for recruiting participants.

This work was supported by the Special Research Fund under Grant number BOF.DC1.2016.0025.01.

No potential conflict of interest was reported by the authors. 


\section{Adaptive Emotion Regulation, Academic Performance, and Internalising Problems in Flemish Children with Special Educational Needs: A Pilot Study}

School readiness of children with special educational needs (SEN) is still understudied. The present study examined how the presumed bidirectional relationship between impaired academic performance (AP) and internalising problems (IP) could be favourably influenced. In this regard, it was assumed that children's adaptive emotion regulation plays a crucial role, as it was shown to be independently related to improved AP and fewer IP. However, to gain stronger evidence for this assumption, it should be further clarified whether adaptive emotion regulation, AP and IP are also jointly associated and, if so, how this is reflected in children with SEN. To explore these issues, two different models were tested in a cross-sectional pilot including 61 Flemish elementary school children with SEN (39 boys and 22 girls, mean age $=10.0$ years old). Teachers reported on adaptive emotion regulation, AP and IP. The results indicated that AP partially mediated the relationship between adaptive emotion regulation and IP (model 1), while IP fully mediated the relationship between adaptive emotion regulation and AP (model 2). Practical implications, strengths, and limitations were discussed.

Keywords: School readiness, Special educational needs, Adaptive emotion regulation, Academic performance, Internalising problems 


\section{Introduction}

\section{Promoting School Readiness in Flemish Children with Special Educational} Needs (SEN)

School readiness, defined by UNICEF as children's successful transition into the school system, implicates specific competencies (e.g. social-emotional and cognitive skills), and is reflected in both psychosocial and academic outcomes (Britto, 2012). Enhancing school readiness is particularly beneficial for young children (i.e. elementary school age) with special educational needs (SEN), as these children have 'learning difficulties or disabilities that make it harder for them to learn or access education than most children of the same age. These children may need extra or different help from that given to other children of the same age' (Westwood, 2007, p. 1).

In Flemish school education (Flemish Ministry of Education, 2017), the largest group of children with SEN consists of children with learning problems and/or a mild intellectual disability, because the group is at specific risk for developing associated psychosocial problems (Dekker, Koot, van der Ende, Verhulst, 2002; Morgan, Farkas, Tufis, \& Sperling, 2008). McIntosh, Ty, and Miller (2014) recommend urgent priority for these internalising problems, as they are assumed to affect academic performances but often remain unaddressed. Academic performance (AP) has been defined as children's achievement, as well as their concrete on-task behaviours (Dupaul, Rapport, \& Perriello, 1991), while internalising problems (IP) refer to 'problems related to anxiety, fear, shyness, low self-esteem, sadness, and depression' (Ollendick \& King, 1994, p. 918).

Taking into account the above recommendations, the present study aimed to explore how the relationship between impaired academic performance and internalising problems could be favourably influenced in children with SEN. In this regard, adaptive 
emotion regulation was assumed to be a crucial mechanism, as it was found to be independently related to improved academic outcomes and fewer internalising problems (Aldao, Nolen-Hoeksema, \& Schweizer, 2010; Bauminger \& Kimhi-Kind, 2008; Graziano, Reavis, Keane, \& Calkins, 2007; MccLure, Halpern, Wolper, \& Donahue, 2009). However, regardless of these findings, this assumption needs to be further substantiated, as it currently remains unclear whether adaptive emotion regulation, academic performance, and internalising problems are also jointly associated and, if so, how these associations are expressed in children with SEN. To address this gap in knowledge, a pilot study was conducted.

\section{Bidirectional Relationship between Academic Performance and Internalising Problems}

In previous research, it has been assumed that AP and IP are bidirectionally related, which resulted in two research lines. Firstly, both cross-sectional and longitudinal studies investigated whether IP precede AP (see e.g. Riglin, Petrides, Frederickson, \& Rice, 2014), which is the adjustment erosion hypothesis (Moilanen, Shaw, \& Maxwell, 2010). According to this hypothesis, higher levels of IP mainly affect students' academic productivity, which is reflected in lower levels of motivation (e.g. striving for rewards), cognitive difficulties (e.g. impaired working memory capacity), and biological complications (e.g. loss of energy) (Weidman, Augustine, Murayama, \& Elliot, 2015). Secondly, mainly longitudinal studies investigated whether low AP precedes IP (see e.g. Moilanen et al., 2010), which is the academic incompetence hypothesis. This hypothesis implies that children with low AP receive negative feedback from their parents and teachers, which subsequently triggers negative primary emotions such as anger, anxiety, and sadness (Cicchetti, Ackerman, \& Izard 1995) leading to IP. 


\section{Promoting Successful School Transition: Emotion Regulation Strategies}

In previous research, one skill that was assumed to individually precede and influence both AP and IP, is emotion regulation (Aldao et al., 2010; Graziano et al., 2007), which has been defined as 'processes by which individuals influence which emotions they have, when they have them, and how they experience and express these emotions' (Gross, 1998, p. 224). When confronted with stressful life-events, such as challenging school demands, children experience both positive and negative emotions. To manage these emotions, children use adaptive and/or maladaptive emotion regulation strategies, based on their positive or negative influence on children's emotions, cognitions, and behaviours (Aldao et al., 2010; Graziano et al., 2007). The present study mainly focuses on adaptive emotion regulation, as firstly, Aldao et al., 2010 showed that adaptive emotion regulation protects children from developing psychopathology, such as internalising problems. Secondly, adaptive emotion regulation has also been associated with improved academic performance (see e.g. Graziano et al., 2007; Juntorn, Sriphetcharawut, \& Munketvit, 2017). Since adaptive emotion regulation seems to be trainable, studies in children with IP were important for designing interventions.

\section{Adaptive Emotion Regulation in the Bidirectional Associations between Academic Performance and Internalising Problems}

Considering the alternative hypotheses on the associations between AP and IP, it remains unclear in which model and how adaptive emotion regulation fits well. First, concerning the academic incompetence hypothesis, one study evaluated the impact of emotion regulation on the assumed AP-IP association but failed to show that AP mediates the association between adaptive emotion regulation and IP (Wills, Simons, Sussman, \& Knight, 2016). Remarkably, also no relationship was observed between adaptive emotion regulation and IP, which is surprising as this was firmly established in 
other research on this topic (see e.g. Eisenberg, Spinrad, \& Eggum, 2010). However, as this study was conducted in a general school population consisting of young adolescents between 11 and 14 years of age, it is difficult to generalize these findings to children with SEN.

Furthermore, when considering emotion regulation, IP, and AP simultaneously and taking into account the adjustment erosion hypothesis, we were unable to find any studies that examined whether low adaptive emotion regulation strategies precede higher levels of IP, which in turn leads to lower levels of AP.

\section{The Current Study}

Based on the above literature review, it can be concluded that there is a gap in knowledge regarding the joint associations between emotion regulation, AP and IP. Therefore, a pilot study was conducted with the main objective to explore how these associations are reflected in elementary school children with SEN using two mediation models. Firstly, it is aimed to tentatively explore whether AP mediates the relation between adaptive emotion regulation and IP in children with SEN, thereby taking into account the academic incompetence hypothesis (Model 1; see Figure 1). Three hypotheses were tested. It was expected that (a) emotion regulation is negatively related to IP and positively to AP, (b) AP is negatively related to IP, and (c) AP mediates the relation between adaptive emotion regulation and IP.

Secondly, it is intended to examine if IP are a mediator in the relationship between adaptive emotion regulation strategies and AP (Model 2; see Figure 2), thereby taking into account the adjustment erosion hypothesis. Again, three hypotheses were tested. It was expected that (a) adaptive emotion regulation is positively associated with AP and negatively with IP, (b) IP are negatively related to AP, and (c) IP mediate the relation between adaptive emotion regulation and AP. 


\section{Method}

\section{Participants}

One Flemish elementary school, taking part in a larger intervention study (Weymeis, Braet, \& Van Leeuwen, 2017), and providing special education for children between 6 and 12 years of age with SEN ('Type Basic Offer', which was defined as education for children with SEN, for whom the common curriculum is not feasible in regular education, unless adjustments; Flemish Ministry of Education, 2017), was included in the current cross-sectional pilot study. The sample size was not predetermined as it was an opportunity sample. A full description of the sample is provided in Table 1. All children between 8 and 12 years of age, as well as their teachers and parents, were invited to participate. Children, parents, and teachers who did not give their explicit consent to participate were removed from the study. Eventually, out of 136 children, a total of 79 teachers, children and their parents agreed to participate. One outlier was excluded from the study $(n=1 ; z-$ score $>3.0)$. Furthermore, as Little's MCAR test showed that the data was not missing at random, $\chi^{2}(9, N=79)=24.10, p=<.05$, participants with too much missing data were excluded from the study using listwise deletion $(n=17)$ (Little, 1988). As a result, the final sample consisted of 61 children (39 boys and 22 girls, mean age $=10.0$ years old), who were all of Caucasian ethnicity. At the time of data collection, children's average time of placement was 2.68 years. To be able to accurately identify children with learning problems (i.e. IQ score $>70$ ) and/or a mild intellectual disability (i.e. IQ score $\geq 55$ and $\leq 70$ ) (American Psychiatric Association, 2013), children's full scale intelligence scores $($ mean $=77.08, S D=11.88)$ were derived from one of three different intelligence tests, which were conducted by the participating school: the Snijders-Oomen Non-Verbal Intelligence test - R (22.60\%) (SON-R; Laros \& Tellegen, 1991), the Wechsler Intelligence Scale for Children - III 
(40.30\%) (WISC-III; Kort et al., 2002) and the Wechsler Preschool and Primary Scale of Intelligence - R (35.50\%) (WPPSI-R; Vander Steene \& Bos, 1997). These tests were equally standardized $($ mean $=100.0, S D=15.0)$ and, moreover, were found to be strongly interrelated (Tellegen, Winkel, Wijnberg-Williams, \& Laros, 1998). As such, it was reasonable to assume that children's intelligence scores can be reliably compared with each other. When comparing the study-subsample with the total school sample, we found that gender, $\chi^{2}(1, N=140)=.05, p=.82$, and socio-economic status (SES), $\chi^{2}(3$, $N=140)=1.98, p=.58$, were not differently distributed. Furthermore, also no significant effect was found regarding age, $t(60)=-1.32, p=.19$ and IQ scores, $t(60)=$ $1.02, p=.31$

[TABLE 1 near here]

\section{Procedure}

The current study was approved by the ethical committee of Ghent University (Ghent University, 2014). Teachers, children, and their parents received a letter including the aim of the study, an invitation to participate, as well as information regarding the confidentiality of data processing. After permission was given, teachers were assigned randomly generated passwords, which provided access to an online survey tool. In this tool, teachers were consequently requested to complete three questionnaires, which are further described below. To avoid the results being affected by ongoing school interventions, all questionnaires were completed at baseline (i.e. at the beginning of the school year). 


\section{Measures}

\section{Emotion Regulation Strategies}

To measure children's use of emotion regulation strategies, the Fragebogen zur Erhebung der Emotionsregulation bei Kindern and Jugendlichen was used (FEEL-KJ; Grob \& Smolenski, 2005; Dutch version by Braet, Cracco, \& Theuwis, 2013). The FEEL-KJ is a 90-item self-, parent- and - teacher report questionnaire for measuring a broad range of emotion regulation strategies in children and adolescents between 8 and 18 years of age. More specifically, the FEEL-KJ assesses 15 emotion regulation strategies in children's response to anxiety, sadness, and anger. Adaptive emotion regulation strategies consist of cognitive problem-solving, problem-solving, acceptance, forgetting, distraction, revaluation, and evoking positive mood. Each strategy is measured by rating two items for each of the three emotions, whereby answers are given on a 5 -point Likert scale $(1=$ almost never; $2=$ rarely; $3=$ occasionally; $4=$ often; $5=$ almost always). Regarding psychometric qualities, the FEEL-KJ is well-validated and reliable (Cracco, Van Durme, \& Braet, 2015). As the current study aims to include a broad range of adaptive emotion regulation strategies, a global composite score of teacher-rated adaptive emotion regulation was used (42 items; $\alpha=.97$ ).

\section{Academic performance}

Students' AP was measured with the Academic Performance Rating Scale (APRS; Dupaul et al., 1991). The APRS is a 19-item teacher questionnaire that assesses children's academic success, academic productivity, and impulse control. The academic success subscale consists of items that are related to children's academic achievement (e.g. quality of reading, quality of speaking, etc.), whereas the academic productivity subscale consists of items related to children's learning behaviours (e.g. time to 
complete work, follows group instructions, etc.). Furthermore, the impulsivity subscale includes items that refer to children's difficulties to work in a precise manner (e.g. begins work carelessly, careless work completion, etc.). Regarding psychometric qualities, the APRS is a valid and reliable instrument for measuring AP (Dupaul et al., 1991). As the current study is especially focused on promoting positive academic outcomes, only academic success ( 9 items; $\alpha=.74)$ and academic productivity ( 7 items; $\alpha=.86)$ were included.

\section{Internalising and Externalising problems}

The Teacher Report Form (TRF; Achenbach \& Rescorla, 2001; Dutch version by Verhulst, Van der Ende, \& Koot, 1997), is a 113-item teacher-report questionnaire for measuring teachers' perceptions of 6- to 18-year-old children's adaptive and maladaptive functioning. Items on the TRF comprise eight subscales, clustered in two broad dimensions, Internalising (Anxious/Depressed, Withdrawn/Depressed, Somatic Complaints) and Externalising (Rule-Breaking Behaviour, Aggressive Behaviour) scales, and a Total Problems scale. Regarding psychometric qualities, the TRF is wellvalidated and reliable (Achenbach, Dumenci, \& Rescorla, 2003). In the current study, children's scores for IP were included as the outcome variable (33 items; $\alpha=.87$ ), whereas externalising problems were included as control variable (33 items; $\alpha=.94$ ).

\section{Data Analytic Plan}

In order to meet the assumption of normality, scores on IP were log-transformed due to positive skewness in the sample distribution, $D(61)=0.21, \mathrm{p}<.05$. Next, descriptive statistics and correlations were calculated (see Table 1). To test the hypothesised mediation models 1 and 2, the SPSS PROCESS macro command (Template 4) was used, which was developed by Preacher and Hayes (2008). The SPSS PROCESS macro 
command involves a nonparametric procedure, which is based on a bias-corrected bootstrap method with 5000 resamples to estimate the $95 \%$ confidence intervals $(\mathrm{CI})$ for the indirect path. It was shown that the bootstrap CI is less susceptible to outliers in small samples compared to other tests (Creedon \& Hayes, 2015). Furthermore, the bootstrapping procedure also seems advantageous over other approaches, as it does not require distributional assumptions and, moreover, takes into account correlations between mediators and control variables. Given these advantages, only pure mediation effects are reported, which are not influenced by other mediators or control variables (Preacher \& Hayes, 2008). In the current study, a .05 criterion for rejection was used.

\section{Results}

\section{Preliminary Analyses}

Descriptive statistics and Pearson correlations are presented in Table 2. As expected, the results showed significant correlations between adaptive emotion regulation and IP, adaptive emotion regulation and AP, and AP and IP. Regarding AP, however, significant correlations were only found for academic productivity. Academic success was consequently excluded as a possible mediating variable in Model 1 and as a possible outcome variable in Model 2. However, as the academic success and productivity scales are typically highly correlated, academic success was included as a control variable in the analyses (see e.g. Graziano et al., 2007). Furthermore, in both models, we controlled for gender, as it is known from the literature that girls typically experience more IP compared to boys (Crijnen, Achenbach, \& Verhulst, 1997). Also children's externalising problems were controlled for, as they commonly interfere with children's IP (Masten et al., 2005).

[TABLE 2 near here] 


\section{Academic Productivity Mediating the Relation between Adaptive Emotion}

\section{Regulation and Internalising Problems}

Regarding Model 1 (see Figure 1), the results confirmed the first (i.e. $c$-path; $\beta=-.01$, $t(56)=-2.93, p<.01$ ), second (i.e. $a$-path; $\beta=.05, t(56)=2.09, p<.05)$, and third hypothesis (i.e. $b$-path; $\beta=-.03, t(55)=-2.52, p<.05$ ). Furthermore, the indirect effect (i.e. $a b-$ path) was estimated between -.004 and -.0003 . As the $95 \%$ confidence interval did not include zero, the results indicated a significant mediation effect. The direct effect remained significant, which suggested partial mediation. Also the final model was significant, $F(4,56)=8.09, p<.001, r^{2}=.01$, explaining $61 \%$ of the variance in IP $\left(R^{2}=\right.$ $\left..61, \Delta R^{2}=.37\right)$.

[FIGURE 1 near here]

\section{Internalising Problems Mediating the Relation between Adaptive Emotion Regulation and Academic Productivity}

Regarding Model 2 (see Figure 2), the assumption was tested that IP mediate the association between adaptive emotion regulation and academic productivity. The results provided support for the first ( $c$-path; $\beta=.05, t(56)=2.09, p<.05)$, second $(a$-path; $\beta=$ $-.01, t(56)=-2.93, p<.01)$ and third hypothesis $(b$-path; $\beta=-3.82, t(55)=-2.52, p<$ .05). Finally, estimations showed that the indirect effect (i.e. $a b-$ path) had a value between .01 and .06 . As the $95 \%$ confidence interval did not include zero, the results indicated a significant mediation effect. The direct effect was no longer significant, which suggested complete mediation. The final model was significant, $F(4,56)=42.36$,

$p<.001, r^{2}=.05$, explaining $87 \%$ of the variance in academic productivity $\left(R^{2}=.87, \Delta\right.$ $\left.R^{2}=.75\right)$.

[FIGURE 2 near here] 


\section{Discussion}

The current pilot study on school readiness aimed to tentatively explore whether and how adaptive emotion regulation, in children with SEN, influenced AP and IP, taking into account the underlying assumption that AP and IP are bidirectionally related (Moilanen et al., 2010). In this regard, one previous study used a non-representative sample (i.e. adolescents without SEN) and failed to show that adaptive emotion regulation precedes the relation between AP and IP (Wills et al., 2016). Conversely, until now, it has remained unclear whether adaptive emotion regulation precedes the relation between IP and AP. To address the above gaps in knowledge, both mediational models, as well as the associated hypotheses, were tested in children with SEN. From the results, and somewhat in line with the academic incompetence hypothesis, it was concluded that the relation between adaptive emotion regulation and IP is partially mediated by children's academic performance (cf. Model 1). Moreover, and slightly in line with the academic erosion hypothesis, it was concluded that the relation between adaptive emotion regulation an academic performance seemed to be entirely mediated by children's IP (cf. Model 2).

Unfortunately, the above analyses do not allow for causal inferences due to the cross-sectional nature of the data. Furthermore, other important limitations prevented us from generalizing any results to a larger population (see also Parker, 1990), such as non-random sampling, the use of a small sample, teacher subjectivity, teacher bias related to difficulties with identifying internalising problems, mono-informant bias, and shared method variance (De Los Reyes \& Kazdin, 2005; McIntosh et al., 2014; Meissel, Meyer, Yao, \& Rubie-Davies, 2017). As a result, it is too early to draw any practical conclusions for schools and teachers at this stage of research. Therefore, this study should be replicated in the future by using a longitudinal design and by including a 
larger sample, different informants, and other data sources such as interviews, observations and test results.

Despite the above limitations, reliable measures were used and, moreover, the study was performed in a real-life setting, thereby guaranteeing richer data and ecological validity (Schmuckler, 2001). Also, as teachers are the primary sources of children's behaviour ratings within the school environment, they are expected to accurately assess children's emotion regulation strategies within an academic context (Evans, Fite, Hendrickson, Rubens, \& Mages, 2015). Overall, the current pilot study provided relevant data that fuel our theoretical understanding of school readiness and offer interesting implications for designing interventions.

The present preliminary findings encourage future research on this topic, which hopefully results in more robust findings and useful indications for teachers and schools to accurately support children with SEN in the classroom.

\section{References}

Achenbach, T. M., L. Dumenci, and L. A. Rescorla. 2003. "DSM-oriented and empirically based approaches to constructing scales from the same item pools." Journal of Clinical Child and Adolescent Psychology 32 (3):328-40. doi:10.1207/S15374424jccp3203_02.

Achenbach, T. M., and L. Rescorla. 2001. Manual for the ASEBA school-age forms \& profiles: an intergrated system of mult-informant assessment. Brulington: VT: University of Vermont, Research Center for Children, Youth, \& Families.

American Psychiatric Association. 2013. Diagnostic and statistical manual of mental disorders (5th ed.). Washington, DC: Author.

Aldao, A., S. Nolen-Hoeksema, and S. Schweizer. 2010. "Emotion-regulation strategies across psychopathology: A meta-analytic review." Clinical Psychology Review 30 (2):217-37. doi:10.1016/j.cpr.2009.11.004.

Bauminger, N., and I. Kimhi-Kind. 2008. "Social information processing, security of attachment, and emotion regulation in children with learning disabilities." 
Journal of Learning Disabilities 41 (4):315-32. doi:

$10.1177 / 0022219408316095$.

Braet, C., Cracco, E., and L. Theuwis. 2013. FEEL-KJ: vragenlijst over emotieregulatie bij kinderen en jongeren. Amsterdam: Hogrefe.

Britto, P. R. . 2012. "School readiness: A conceptual framework." In United Nations Children's Fund, edited by United Nations Children's Fund. New York: United Nations Children's Fund.

Cicchetti, D., B. P Ackerman, and C. E Izard. 1995. Emotions and emotion regulation in developmental psychopathology. Development and psychopathology, 7(1), 1-10.

Cracco, E., K. Van Durme, and C. Braet. 2015. "Validation of the FEEL-KJ: an instrument to measure emotion regulation strategies in children and adolescents (vol 10, e0137080, 2015)." Plos One 10 (12). doi:ARTNe014432410.1371/journal.pone.0144324.

Creedon, P.S., and A. F. Hayes. 2015. "Small sample mediation analysis: How far can you push the bootstrap?" In Annual conference of the Association for Psychological Science. New York.

Crijnen, A. A. M., T. M. Achenbach, and F. C. Verhulst. 1997. "Comparisons of problems reported by parents of children in 12 cultures: Total problems, externalizing, and internalizing." Journal of the American Academy of Child and Adolescent Psychiatry 36 (9):1269-77. doi:10.1097/00004583-19970900000020.

De Los Reyes, A., and A. E. Kazdin. 2005. "Informant discrepancies in the assessment of childhood psychopathology: A critical review, theoretical framework, and recommendations for further study." Psychological Bulletin 131 (4):483-509. doi:10.1037/0033-2909.131.4.483.

Dekker, M. C., H. M. Koot, J. van der Ende, and F. C. Verhulst. 2002. "Emotional and behavioral problems in children and adolescents with and without intellectual disability." Journal of Child Psychology and Psychiatry and Allied Disciplines 43 (8):1087-98. doi:10.1111/1469-7610.00235.

Dupaul, G. J., M. D. Rapport, and L. M. Perriello. 1991. "Teacher ratings of academic skills - the development of the Academic-Performance Rating-Scale." School Psychology Review 20 (2):284-300. 
Eisenberg, N., T. L. Spinrad, and N. D. Eggum. 2010. "Emotion-related self-regulation and its relation to children's maladjustment." Annual Review of Clinical Psychology, Vol 6 6:495-525. doi:10.1146/annurev.clinpsy.121208.131208.

Evans, S. C., P. J. Fite, M. L. Hendrickson, S. L. Rubens, and A. K. Mages. 2015. "The role of reactive aggression in the link between hyperactive-impulsive behaviors and peer rejection in adolescents." Child Psychiatry \& Human Development 46 (6):903-12. doi:10.1007/s10578-014-0530-y.

Flemish Ministery of Education. 2017. "Statistical Yearbook of Flemish Education." In, 1-582. Brussels: Flemish Department of Education.

Ghent University. 2014. "Ethical approval: Back 2 Forward: the relation between a school-based Time-In programme and social-psychological functioning of primary school children." Ghent: Ghent University.

Graziano, P. A., R. D. Reavis, S. P. Keane, and S. D. Calkins. 2007. "The role of emotion regulation in children's early academic success." Journal of School Psychology 45 (1):3-19. doi:10.1016/j.jsp.2006.09.002.

Grob, A., and C. Smolenski. 2005. Fragebogen zur Erhebung der Emotionsregulation bei Kindern und Jugendlichen. Bern: Huber.

Gross, J. J. 1998. "Antecedent- and response-focused emotion regulation: Divergent consequences for experience, expression, and physiology." Journal of Personality and Social Psychology 74 (1):224-37. doi:10.1037//00223514.74.1.224.

Juntorn, S., S. Sriphetcharawut, and P. Munketvit. 2017. "The relationship between information processing strategies application and social competence in Thai children with learning disabilities." Journal of Associated Medical Sciences 50 (1):38-53.

Kort, W., M. Schittekatte, E. L. Compaan, M.Bosmans, N. Bleichrodt, G.Vermeir, ... and P. Verhaeghe. 2002. WISC-III-NL: Manual Dutch adaptation. The Psychological Corporation, London.

Laros, J. A., P. J. Tellegen, and B. J. Wijnberg-Williams. 1991. Construction and Validation of the SON-R 5-1/2-17, the Snijders-Oomen Non-verbal Intelligence Test. Groningen, The Netherlands: Wolters-Noordhoff.

Little, R. J. A. 1988. "A test of missing completely at random for multivariate data with missing values." Journal of the American Statistical Association 83 (404):1198202. doi:10.2307/2290157. 
Masten, A. S., G. I. Roisman, J. D. Long, K. B. Burt, J. Obradovic, J. R. Riley, K. Boelcke-Stennes, and A. Tellegen. 2005. "Developmental cascades: Linking academic achievement and externalizing and internalizing symptoms over 20 years." Developmental Psychology 41 (5):733-46. doi:10.1037/00121649.41.5.733.

MccLure, K.S., J. Halpern, P. A. Wolper, and J. Donahue. 2009. "Emotion regulation and intellectual disability." Journal of Developmental Disabilities 15 (2):38-44.

McIntosh, K., S. V. Ty, and L. D. Miller. 2014. "Effects of school-wide positive behavioral interventions and supports on internalizing problems: current evidence and future directions." Journal of Positive Behavior Interventions 16 (4):209-18. doi:10.1177/1098300713491980.

Meissel, K., F. Meyer, E. S. Yao, and C. M. Rubie-Davies. 2017. "Subjectivity of teacher judgments: Exploring student characteristics that influence teacher judgments of student ability." Teaching and Teacher Education 65:48-60. doi: 10.1016/j.tate.2017.02.021.

Moilanen, K. L., D. S. Shaw, and K. L. Maxwell. 2010. "Developmental cascades: Externalizing, internalizing, and academic competence from middle childhood to early adolescence." Development and Psychopathology 22 (3):635-53. doi:10.1017/S0954579410000337.

Morgan, P. L., G. Farkas, P. A. Tufis, and R. A. Sperling. 2008. "Are reading and behavior problems risk factors for each other?" Journal of Learning Disabilities 41 (5):417-36. doi:10.1177/0022219408321123.

Ollendick, T. H., and N. J. King. 1994. "Diagnosis, assessment, and treatment of internalizing problems in children - the role of longitudinal data." Journal of Consulting and Clinical Psychology 62 (5):918-27. doi:10.1037/0022006x.62.5.918.

Parker, R. M. 1990. "Power, control, and validity in research." Journal of Learning Disabilities 23 (10):613-20.

Preacher, K. J., and A. F. Hayes. 2008. "Asymptotic and resampling strategies for assessing and comparing indirect effects in multiple mediator models." Behavior Research Methods 40 (3):879-91. doi:10.3758/Brm.40.3.879.

Riglin, L., K. V. Petrides, N. Frederickson, and F. Rice. 2014. "The relationship between emotional problems and subsequent school attainment: A meta- 
analysis." Journal of Adolescence 37 (4):335-46.

doi:10.1016/j.adolescence.2014.02.010.

Schmuckler, M. A. 2001. "What is ecological validity? A dimensional analysis." Infancy 2 (4):419-36. doi:10.1207/S15327078in0204_02.

Tellegen, P.J., M. Winkel, B.J. Wijnberg-Williams, and J.A. Laros. 1998. SnijdersOomen Nonverbal Intelligence Test. SON-R 21/2-7 Manual and Research Report. Lisse: Swets \& Zeitlinger B.V.

Vander Steene, G., and A. Bos. 1997. WPPSI-R. Wechsler Preschool and Primary Scale of Intelligence - Revised. Nederlandstalige bewerking. Amsterdam: Paerson.

Verhulst, F. C., J. Van der Ende, and H.M. Koot. 1997. Dutch Manual for the Teacher's Report Form (TRF). Rotterdam: Afdeling Kinder-en Jeugdpsychiatrie, Sophia. Kinderziekenhuis/Academisch Ziekenhuis Rotterdam, Erasmus Universiteit Rotterdam.

Weidman, A. C., A. A. Augustine, K. Murayama, and A. J. Elliot. 2015. "Internalizing symptomatology and academic achievement: Bi-directional prospective relations in adolescence." Journal of Research in Personality 58:106-14. doi:10.1016/j.jrp.2015.07.005.

Westwood, P. 2007. Commonsense methods for children with special educational needs. New York: Routledge.

Weymeis, H., C. Braet, and K. Van Leeuwen. 2017. "Extending School-Wide Positive Behavior Support (SWPBS) with Emotional Support Systems in Promoting School Readiness: Effect of TIME-IN on Special Education Children's School Readiness." Manuscript in preparation.

Wills, T. A., J. S. Simons, S. Sussman, and R. Knight. 2016. "Emotional self-control and dysregulation: A dual-process analysis of pathways to externalizing/internalizing symptomatology and positive well-being in younger adolescents." Drug and Alcohol Dependence 163:S37-S45. doi:10.1016/j.drugalcdep.2015.08.039. 
Table 1. Sample Table for Describing Participants

\section{Participants}

Gender:

male

female

total

Age:

mean

range

Race/Ethnicity:

Caucasian

SES:

upper class

upper-middle class

middle class

lower-middle class

Level of Placement:

Average time in Special Ed. Placement

Level of Placement

Intelligence*:

IQ score $\leq 70$

IQ score $>70$

mean

range $n=39(63.90 \%)$

$n=22(36.10 \%)$

$n=61$

$4.0(8.0-12.0)$

$n=61$

$n=1(1.60 \%)$

$n=14(23.0 \%)$

$n=35(57.40 \%)$

$n=11(18.0 \%)$

2.68

Type Basic Offer

$n=16(26.20 \%)$

$n=45(73.80 \%)$

77.08

$55.0(55.0-110.0)$

Overall Academic Achievement**: 
mean

$S D$

range
22.13

4.11

$20.0(12.0-32.0)$

Urban

Urban

Geographical location:

Note. $^{*}=$ Names of tests used: SON-R, WISC-III and WPPSI-R; $* *=$ Name of test

used: APRS (Academic Success scale). 
Table 2. Study Variables Pearson Correlations and Descriptive Statistics

\begin{tabular}{|c|c|c|c|c|c|c|c|c|c|}
\hline Variable & 1 & 2 & 3 & 4 & 5 & $M$ & $S D$ & Min & Max \\
\hline 1. Adaptive & & & & & & 112.28 & 23.27 & 57.0 & 166.0 \\
\hline \multicolumn{10}{|l|}{ emotion regulation } \\
\hline 2. Academic success & .14 & & & & & 22.13 & 4.11 & 12.0 & 32.0 \\
\hline 3. Academic & $.25^{*}$ & $.86^{* *}$ & & & & 40.21 & 7.55 & 20.0 & 57.0 \\
\hline \multicolumn{10}{|l|}{ productivity } \\
\hline 4. Internalising & $-.46 * *$ & .16 & $-.35 * *$ & & & 6.18 & 6.32 & 0.0 & 27.0 \\
\hline \multicolumn{10}{|l|}{ problems } \\
\hline 5. Age & -.08 & -.09 & -.09 & .18 & & 10.0 & 1.37 & 8.0 & 12.0 \\
\hline 6. IQ score & -.15 & -.019 & -.04 & -.001 & -.09 & 77.08 & 11.88 & 55.0 & 110.0 \\
\hline
\end{tabular}

Note. ${ }^{*} p<.05 ; * * p<.01$. Min $=$ Minimum; Max $=$ Maximum. 
Figure 1. Mediation Model 1

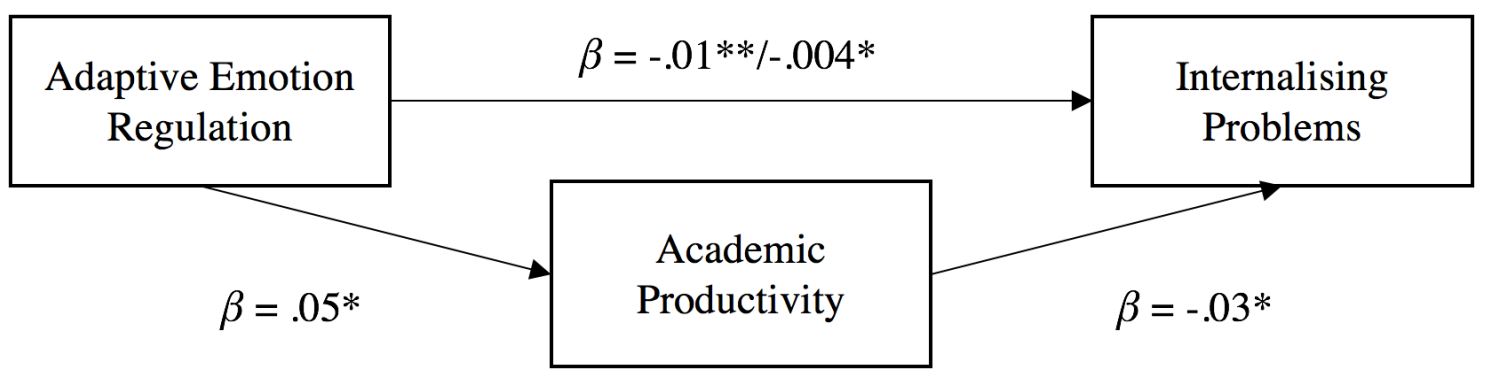

Figure 1. Academic productivity as a mediator between adaptive emotion regulation and internalising problems.

Note. ${ }^{*} p<.05 ; * * p<.01$. 
Figure 2. Mediation Model 2

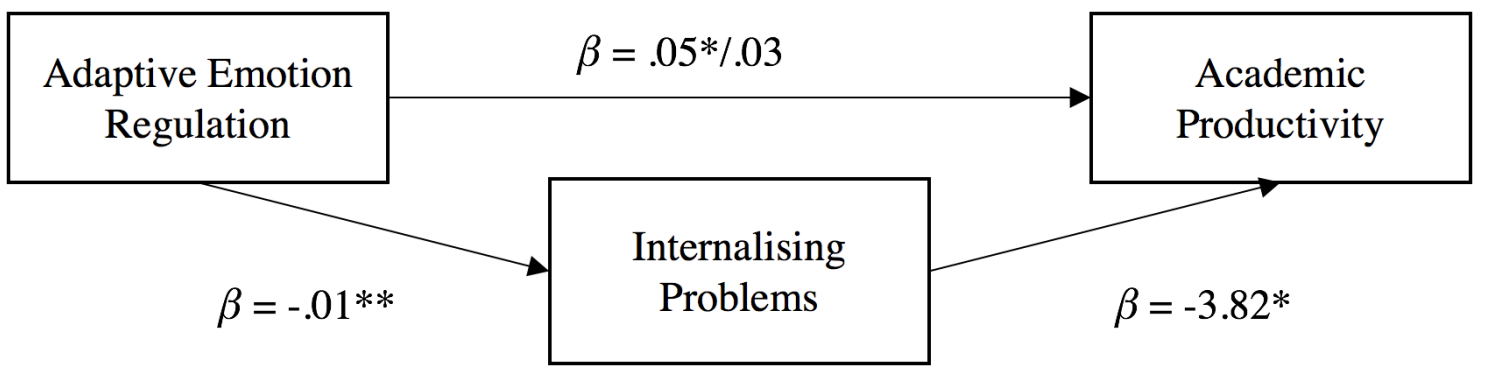

Figure 2. Internalising problems as a mediator between adaptive emotion regulation and academic productivity.

Note. ${ }^{*} p<.05 ; * *<.01$. 